International Journal of Emerging Trends in Engineering Research

Available Online at http://www.warse.org/IJETER/static/pdf/file/ijeter517112019.pdf

https://doi.org/10.30534/ijeter/2019/517112019

\title{
Some Features in Calculation of Mold Details for Plastic Products
}

\author{
Vyacheslav Lyashenko $^{1}$, Syed Khalid Mustafa ${ }^{2}$, Nataliya Belova ${ }^{3}$, M. Ayaz Ahmad ${ }^{4}$ \\ ${ }^{1}$ Department of Informatics, Kharkiv National University of RadioElectronics, Ukraine, \\ lyashenko.vyacheslav@gmail.com \\ ${ }^{2}$ Department of Chemistry, Faculty of Science, University of Tabuk, Saudi Arabia, \\ khalid.mustafa938@gmail.com \\ ${ }^{3}$ Department of Informatics, Kharkiv National University of RadioElectronics, Ukraine, \\ nataliia.bielova@nure.ua \\ ${ }^{4}$ Department of Physics, Faculty of Science, University of Tabuk, Saudi Arabia, mayaz.alig@gmail.com
}

\begin{abstract}
The article considers the relationship of mold details and injection molds. The basic elements of mold details are considered. The calculation method of design data tooling which can be used at design of injection mold is offered. This method gives the chance to provide the necessary accuracy of casting. This allows to determine the rational walls thickness of the injection mold and to define further what type of heat treatment is necessary after formation.
\end{abstract}

Key words: Injection Mold, Mold Details, Plastic Products, Mold Cavity, Mold Core, Punch, Matrix.

\section{INTRODUCTION}

Injection molds (IM) are the basis for the manufacture of plastic products. The use of this technology allows obtaining large quantities of products at relatively low unit cost [1]. Structurally, the IM consists of two main parts: the punch and the matrix (mold cavity and mold core). System of the mold details (MD) is the foundation of IM [2], [3]. An essential role at design of IM also is played by the system of the mold details, which at the closed semi-forms form the mold cavity (MC) (see Fig. 1).

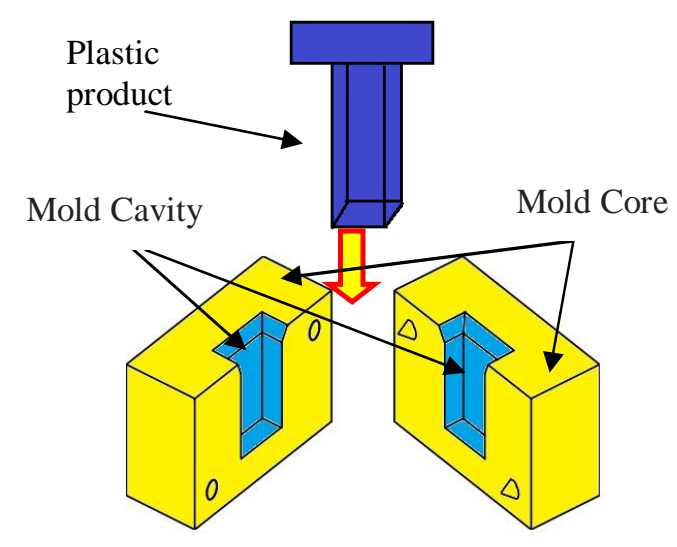

Figure 1: System of the mold details
The details design of system generally is defined by the nature of filling of the mold cavity and directly influences quality of the received plastic product (casting) [4], [5]. Solution of this task can be reached thanks to the MD rational sizes. Therefore, the problem of calculating data occupies an important place in the production process of any products.

\section{REVIEW OF REFERENCES ON A RESEARCHES SUBJECT}

The basic design principles of the tooling for injection under pressure and its design features are put in work of Rees H. [1]. It is possible to find the full creation review and use of IM in this work: since injection-molding machine, the basic principles of products design, forming materials and finishing with management of formation process.

D. V. Rosato and M. G. Rosato are considered by the theoretical information about structure and properties of plastic, methods of their receiving and processing, features of a of injection under pressure method and also the main types of automatic molding machines are considered [6]. Special attention is paid to bases of design and calculation of injection moulds. Authors have given the general calculation method of the executive sizes of injection molds under pressure. In the research conducted by D. V. Rosato and M. G. Rosato a concept the executive size belongs to the sizes of the mold cavity of a form and means the final size of the form element which is mold a plastic detail.

M. R. Kamar represents the comprehensive, balanced combination of the practical and theoretical principles of injection under pressure, including how to project and make own forms [7]. The author has given examples of creation of the first form and has described process of the choice of material for IM; examples of injection gains calculation and also the number of the mold cavities [7].

V. R. Gingery has submitted the stage-by-stage instructions opening secrets of construction of the small inexpensive injection molding machine capable to pressing of the majority 
types of plastic [8]. In [8] are also shown various types of forms and their structure. In the researches V. R. Gingery he has considered prototype forms and analyzed process of form filling. The author has in details analyzed parameters of injection process and the tasks solution of high-quality management of this process. V. R. Gingery has given calculations of the executive sizes of simple punches and matrixes; the guide to design of IM for formation of thermoplastics is provided [8].

Standard IM and its elements, optimization of the mold cavity sizes and the choice of material were in details described by $U$. Bruder [9]. The author has considered features of design of sprue system, the system of pushing out (the main problems of pushing out; details for pushing out; design of elements of pushing out); ventilating system. U. Bruder has carried out the analysis of constructional features of forms for ensuring their durability and durability [9].

T.A. Osswald, L.S. Turng and P.J. Gramann have conducted the last researches in the field of mathematical modeling and optimization of injection under pressure process, have considered optimization methods which include the short description of calculation methods of the matrixes and punches sizes. Authors have presented the modern researches devoted to mathematical modeling of process of IPUP with an explanation of simulations process and advantages of each concrete model [4].

\section{FEATURES OF THE BASIC ELEMENTS OF MD}

Basic elements of MD are punches and matrixes. These elements are one-piece or compound.

Features of one-piece punches and matrixes. Such designs are less reliable (because of their attrition, the shift of axes fusion flowing is possible, bark), but are stronger, resist to external loadings better. In them the pushers cooling channels rather conveniently (if the sizes allow) are placed. In Fig. 2 designs of matrixes are shown. One-piece matrixes apply in the single-gates forms, a thicket - to big products. In the multiple gates forms one-piece matrixes apply for the purpose of possible rapprochement of gates, reduction of the form sizes (if it is necessary, proceeding from the available park of the equipment) [2], [3].

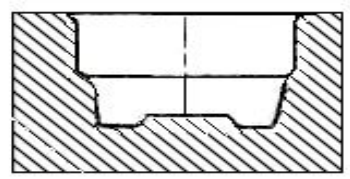

a)

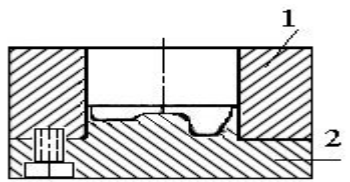

b)

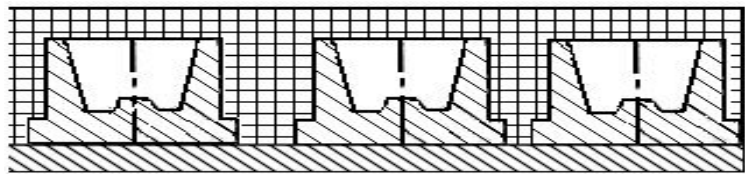

c)

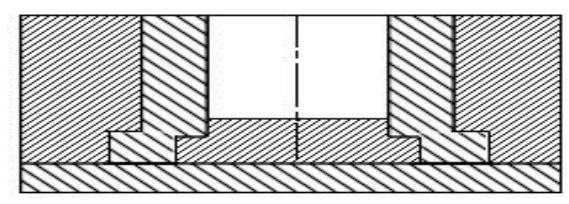

d)

Figure 2: Scheme of a design of matrixes of IM: a) one-piece; b) for pressing of products with a difficult contour; c) composite; d) compound

Kinds of the simplest punches design are presented in Fig. 3.

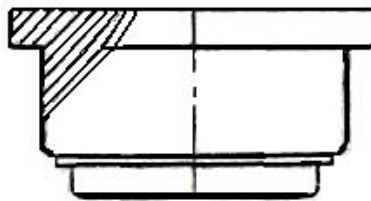

a)

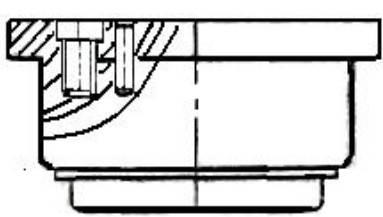

b)

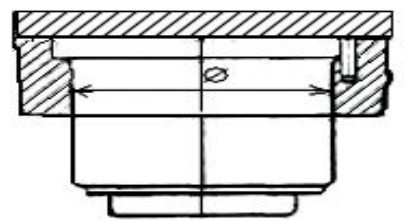

c)

Figure 3: Execution construction scheme of punches designs: a) one-piece punch; b) punch with fastening; c) design of the punch established in a plate-stem holder

Features of compound punches and matrixes. The compound mold details design when it is necessary to make products with undercuts, high edges at rather small thickness of walls, a significant amount of grooves, the openings located both in the direction of the main semi-forms movement and perpendicular to him The variety of construction design connected with a products variety is very high, and the general recommendations come down to the following: elements of compound punches and matrixes have to be strong, and places of their joints - tight [10]-[12]. It is expedient to create the auto fastened designs allowing considering a compound detail as monolithic, but it is allowed to apply also additional fastenings, constructive grooves and fillets, etc. Examples the single-gates and the multiple gates of forms are presented in Fig. 4, a, b [6]. 


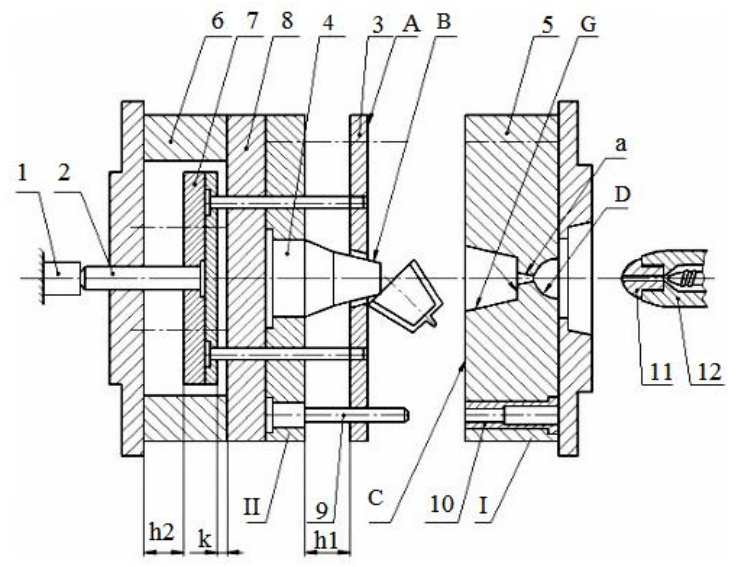

a)

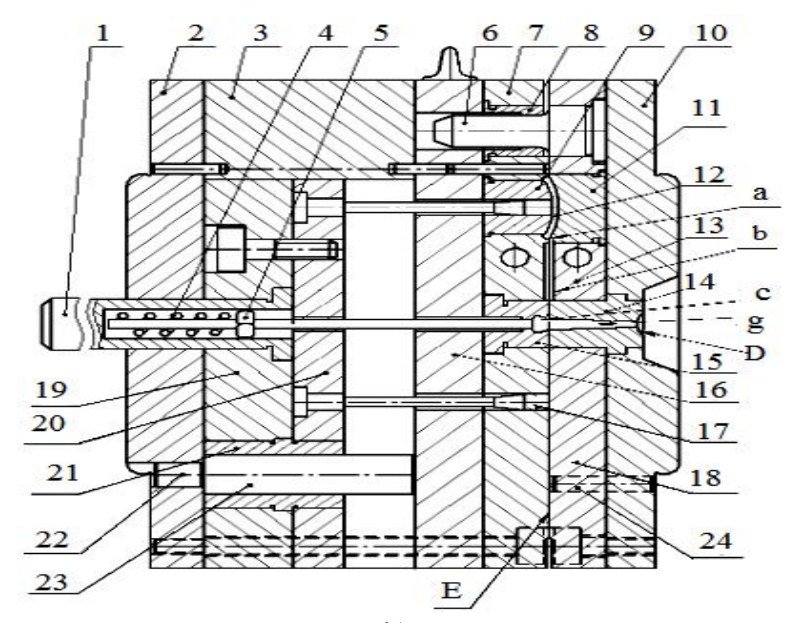

b)

Figure 4: Standard designs of injection molds: a) single-gates form for a "glass" product; b) multiple gates form for a "cover" product

The single-gates injection mold (Fig. 4, a) consists from: I motionless and the II mobile semi-forms; A, B, G - mold cavity; $\mathrm{C}$ - the closing matrix surface; $\mathrm{D}$ - spherical surface of a sprue channel; E - sprue channel; 1 - motionless machines emphasis; 2 - rod; 3 - pushing together plate; 4 - punch; 5 matrix; 6 - bars of the support plate; 8 (top) and 7 (lower) connecting levels; 9 - directing columns; 10 - directing plugs; 11 - nozzle, 12 - gate of the cylinder of the injection molding machines, which at short circuits of a form are interfaced with and align semi-forms.

The multiple gates injection mold (Fig. 4, b) consists from: a - inlet channels; $b$ - parting ingates; $c$ - undercutting opening in the tripper device plug; g- central ingate; D - spherical part of a nozzle; E - the closing matrix holder plane; 1 - rod; 2 - back flange; 3 - centering detail; 4 - spring; 5 - tripper device; 6, 7, 8 - centering details; 9 - punch; 10 - forward flange; 11 - plug-in gates; 12 - cover; 13 - centering detail; 14 - plug; 15 - tripper device; 16 - support plate; 17 returnable pusher; 18 - holders of matrixes; 19, 20 - connecting levels; 21 - directing plug; 22 - column; 23 pusher; 24 - centering detail.

At design of all MD, during development of their working drawings, count such executive sizes, compensating possible fluctuations and changes in their sizes.

\section{CALCULATION OF THE MD EXECUTIVE SIZES}

Between the nominal product size $N_{p}$ and the executive size of the mold detail $N_{m}$, as well as between their admissions respectively $T_{p}$ and $T_{m}$, there is an interrelation. We will review an example when fields of sizes admissions are symmetrized in relation to the sizes: $N_{p} \pm T_{p}$ and $N_{m} \pm T_{m}$.

Under such circumstances the largest size of a product $N_{p}+T_{p}$ can be defined if the size of the mold detail is maximum $N_{m} \pm T_{m}$, and shrinkage of the processed material - minimum $S_{\min }$.

On the other hand, the size of a detail $N_{p}-T_{p}$ can be received in case the formed material has the maximum shrinkage $S_{\text {max }}$, the mold detail is executed by the size $N_{m}-T_{m}$. So, we receive system:

$$
\left\{\begin{array}{l}
\left(N_{m}+T_{m}\right)-\left(N_{m}+T m\right) \cdot S_{\min }=N_{p}+T_{p} \\
\left(N_{m}-T_{m}\right)-\left(N_{m}-T_{m}\right) \cdot S_{\max }=N_{p}-T_{p}
\end{array}\right.
$$

Proceeding from that, as $T_{m} S_{\text {min }}$ and $T_{m} S_{\text {max }}$ are negligible in comparison with $N_{m} S_{\text {min }}$ and $N_{m} S_{\max }$, the system becomes simpler:

$$
\left\{\begin{array}{l}
N_{m}+T_{m}-N_{m} \cdot S_{\text {min }}=N_{p}+T_{p} \\
N_{m}-T_{m}-N_{m} \cdot S_{\text {max }}=N_{p}-T_{p}
\end{array}\right.
$$

Summarizing and entering average shrinkage, we receive the equation connecting the sizes of the mold detail and product with average shrinkage $\bar{S}: N_{m}(1-\bar{S})=N_{p}$.

If to solve the given system concerning admissions, then ratios turn out:

$$
\begin{gathered}
T_{m}=T_{p}-\frac{N_{m}\left(S_{\max }-S_{\min }\right)}{2}=T_{p}-\frac{N_{m} \Delta S}{2} ; \\
\mathrm{T}_{\mathrm{p}}=\mathrm{T}_{\mathrm{m}}+\frac{\mathrm{N}_{\mathrm{m}} \Delta \mathrm{S}}{2} ;
\end{gathered}
$$


Vyacheslav Lyashenko et al., International Journal of Emerging Trends in Engineering Research, 7(11), November 2019,720 - 724

$$
\Delta \mathrm{S}=\mathrm{S}_{\max }-\mathrm{S}_{\min }
$$

In Fig. 5 advantage of the calculation focused on the average element size of a product is shown. Such calculation most fully meets the requirements of mass, large-lot production, problems of precision products release as the average size actually this selective average distribution $\bar{X}$ of the sizes (in a limit - population mean of the probable size with confidential borders $\pm 3 \delta$ within the set tolerance zone). Reliability of such calculation scheme is provided also that most parties of materials is issued with the indicators of coefficients of shrinkage close to $\overline{\mathrm{S}}$.

When calculating the executive sizes of the mold details, we will specify in addition designations:

1) T att.m - the admission on elements attrition of the mold details.

2) The indexes min, max - presented at the corresponding sizes, indicate the maximum, minimum values of these sizes;

3) Indexes of $m$, p belong to a matrix (opening) and a punch (shaft);

4) Indexes sh, about o to a plastic shaft and an opening in a plastic product;

5) The line over size means its average value.

Admissions of a MD product $T_{p}$ and $T_{m}$ are also appointed by designers of the forming tool and a plastic product on finish.

In process of forms attrition at IPUP there is some provision change of the average size $\bar{X}$ that leads to the insignificant shift of the sizes dispersion field.

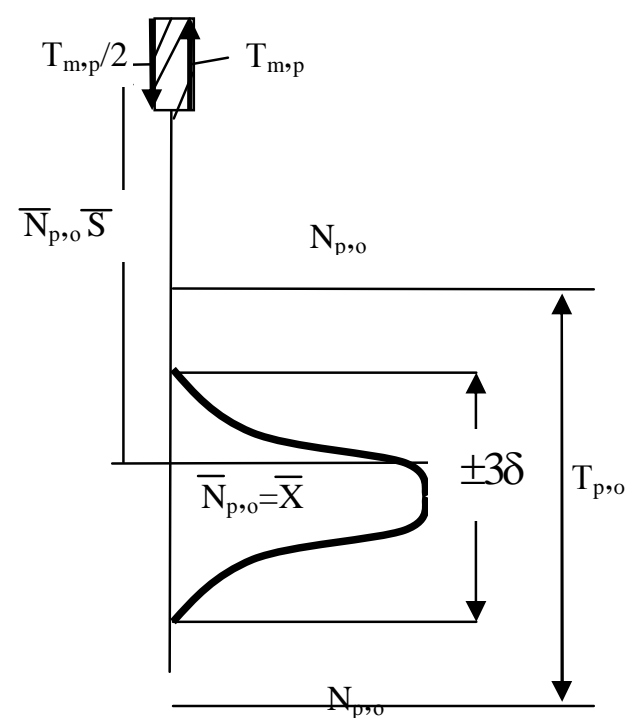

Figure 5: The scheme to calculation of the mold detail (punch) executive size
It is accepted expedient that $T_{\text {att.m }}=T_{\text {att.m }} / 2$ as the basic forming elements of details (matrixes, punches, signs) finally process by a method of "trial passes", operational development on high-precision machines or manually when after the admission $T_{m}$ completely it isn't used, and processing stops, only the size of an element gets to the set tolerance zone, but near the corresponding extreme sizes (the greatest - for a punch, or "shaft"; the smallest - for a matrix, or "opening"); therefore, reserves of the field $T_{m}$ can be used for compensation of attrition.

\section{DURABILITY OF SEPARATE ELEMENTS}

Calculations of durability of a punch and matrixes of forms carry out for determination of rational walls thickness. Calculations of durability are complemented with calculations of especially loaded details deformations.

Rational submission of the forces distribution scheme in relation to a concrete design of pair, "a punch - a matrix" and other details of a form interfaced to her is also necessary. Therefore as an example we will consider the main method of carrying out calculations of deformability and durability of the mold details on the example of one-piece a matrix and the punch forming a cylindrical gate.

The maximum change of nominal wall thickness of a plastic product can be defined on [6].

$$
\delta_{\max }=\delta+\Delta \mathrm{r}_{\mathrm{m}}+\Delta \mathrm{R}_{\mathrm{p}}
$$

where $\delta$-wall thickness of a product is equal $\delta=r_{M}-R_{n}$;

$\Delta r_{m}$ - deformation of the forming matrix surface;

$\Delta R_{p}$ - deformation of the forming punch surface.

But sum $\Delta r_{M}+\Delta R_{n}<2 \delta S(\mathrm{~S}-$ shrinkage, $\mathrm{mm} / \mathrm{min}$.$) .$

To determine sizes $\Delta r_{m}$ and $\Delta R_{p}$ by an distribution of tension arising in a matrix and a punch under the influence of injection pressure. The theorem of Lyames about tangential tension on a surface of a punch and a matrix is applied to tension assessment on MD [4, 6].

The method considered above allows determining the rational walls thickness of a injection mold which has to be rational to avoid internal tension. As it affects mechanical properties and behavior of products at operation. Thus the considered method allows defining further what type of heat treatment is necessary after formation in order that the product had necessary superficial durability in order to avoid defects. 
Vyacheslav Lyashenko et al., International Journal of Emerging Trends in Engineering Research, 7(11), November 2019,720 - 724

\section{CONCLUSION}

Mold details are the foundation of injection molds. It is important to know the dimensions of mold details and injection molds.

This is due to that accuracy and quality of castings depends first of all on the accuracy of production of IM and the MD sizes.

Therefore, we examined the features of the bang elements mold details. Basic elements of MD are punches and matrixes.

For this, we have summarized calculation of the MD executive sizes and durability of separate elements. This allows to determine the rational walls thickness of the injection mold and to define further what type of heat treatment is necessary after formation.

The offered calculation method of design data of the tooling allows increasing the accuracy of the received plastic product.

\section{REFERENCES}

1. H. Rees. Understanding injection mold design, Hanser Publishers, 2001.

2. S. Sotnik, R. Matarneh, and V. Lyashenko. System Model Tooling For Injection Molding, International Journal of Mechanical Engineering and Technology, Vol. 8, no. 9, pp. 378-390, 2017.

3. M. H. Al-Sherrawi, A. M. Saadoon, S. Sotnik, and V. Lyashenko. Information model of plastic products formation process duration by injection molding method, International Journal of Mechanical Engineering and Technology, Vol. 9, no. 3, pp. 357-366, 2018.

4. T. A. Osswald, L. S. Turng, and P. J. Gramann. Injection Molding Handbook, Hanser Verlag, 2002.

5. C. Fernandes, A. J. Pontes, J. C. Viana, and A. Gaspar-Cunha. Modeling and Optimization of the Injection-Molding Process: A Review, Advances in Polymer Technology, Vol. 37, no. 2, pp. 429-449, 2018. https://doi.org/10.1002/adv.21683

6. D. V. Rosato, and M. G. Rosato. Injection molding handbook, Springer Science \& Business Media, 2012.

7. M. R. Kamar. Injection molding: Technology and fundamentals, Cincinnati: Hanser, 2009.

8. V. R. Gingery. The Secrets of Building a Plastic Injection Molding Machine, Publishing: LLC, 2015.

9. U. Bruder. User's Guide to Plastic: A handbook for everyone, Carl Hanser Verlag GmbH \& Co, 2015.

10. I. Ferreira, J. A. Cabral, P. Saraiva, and M. C. Oliveira. A multidisciplinary framework to support the design of injection mold tools, Structural and Multidisciplinary
Optimization, Vol. 49, no. 3, pp.501-521, 2014.

https://doi.org/10.1007/s00158-013-0990-x

11. J. Rotheiser. Joining of plastics: handbook for designers and engineers, Carl Hanser Verlag GmbH Co KG, 2015.

12. M. Piazza, and S. Alexander. Molding: A Summary of the Literature, Urban Publications, 2015. 